\title{
Componentwise Complementary Cycles in Almost Regular 3-Partite Tournaments
}

\author{
Zhihong $\mathrm{He}^{1, \star}$, Guojun $\mathrm{Li}^{1}$ Dawei Ding ${ }^{2}$, and Quanhui Liu ${ }^{3}$ \\ ${ }^{1}$ School of Mathematics and System Sciences, Shandong University, \\ Jinan, 250100, China \\ zhihhe@126.com \\ 2 Opto-Electronic Information, Yantai University, Yantai, 264005, China \\ ${ }^{3}$ College of Mathematics and Information, Ludong University, Yantai, \\ 264005, China
}

\begin{abstract}
The vertex set of a digraph $D$ is denoted by $V(D)$. A $c$ partite tournament is an orientation of a complete $c$-partite graph. Let $V_{1}, V_{2}, \ldots, V_{\mathrm{c}}$ be the partite sets of $D$. If there exist two vertex disjoint cycles $C$ and $C^{\prime}$ in $D$ such that $V_{\mathrm{i}} \cap\left(V(C) \cup V\left(C^{\prime}\right)\right) \neq \emptyset$ for all $i=1,2, \ldots, c$, then $D$ is cycle componentwise complementary. The global irregularity of $D$ is defined by $i_{\mathrm{g}}(D)=\max \left\{\max \left(d^{+}(x), d^{-}(x)\right)-\right.$ $\left.\min \left(d^{+}(y), d^{-}(y)\right) \mid x, y \in V(D)\right\}$ over all vertices $x$ and $y$ of $D(x=y$ is admissible), where $d^{+}(x)$ and $d^{-}(x)$ are the outdegree and indegree of $x$, respectively. If $i_{\mathrm{g}}(D) \leq 1$, then $D$ is almost regular. In this paper, we consider a special kind of multipartite tournaments which are almost regular 3-partite tournaments, and we show that each almost regular 3partite tournament $D$ is cycle componentwise complementary, unless $D$ is isomorphic to $D_{3,2}$.
\end{abstract}

Keywords: almost regular, componentwise complementary cycles, tournament.

\section{Terminology and Preliminary Results}

We shall assume that the reader is familiar with the standard terminology on directed graphs and refer the reader to [1]. In this paper all digraphs are finite without loops or multiple arcs. The vertex set and the $\operatorname{arc}$ set of a digraph $D$ are denoted by $V(D)$ and $E(D)$, respectively. For a vertex set $X$ of $D$, we define $D[X]$ as the subdigraph induced by $X$. If $x y$ is an arc of a digraph $D$, then we write $x \rightarrow y$ and say $x$ dominates $y$.

A $c$-partite or multipartite tournament is an orientation of a complete $c$ partite graph. A tournament is a $c$-partite tournament with exactly $c$ vertices. A semicomplete multipartite digraph is obtained by replacing each edge of a complete multipartite graph by an arc or by a pair of two mutually opposite arcs with the same end vertices. Let $V_{1}, V_{2}, \ldots, V_{c}$ be the partite sets of a $c$-partite tournament or semicomplete $c$-partite digraph $D$ and $\left|V_{1}\right| \leq\left|V_{2}\right| \leq \ldots \leq\left|V_{\mathrm{c}}\right|$. If

\footnotetext{
^ Corresponding author.
} 
the vertex $x$ of $D$ belongs to the partite set $V_{\mathrm{i}}$, then we define $V(x)=V_{\mathrm{i}}$ and define $\gamma(D)=V_{1}$.

If $X$ and $Y$ are two disjoint subsets of $V(D)$ or subdigraphs of $D$ such that every vertex of $X$ dominates every vertex of $Y$, then we say that $X$ dominates $Y$, denoted by $X \rightarrow Y$. If there is no arc from $Y$ to $X$, but $x \rightarrow y$ for all $x \in X$ and $y \in Y$ which are in different partite sets, then we denote this by $X \Rightarrow Y$ which denotes the property that there is no $\operatorname{arc}$ from $Y$ to $X$.

The out-neighborhood $N_{\mathrm{D}}^{+}(x)=N^{+}(x)$ of a vertex $x$ is the set of vertices dominated by $x$, and the in-neighborhood $N_{\mathrm{D}}^{-}(x)=N^{-}(x)$ is the set of vertices dominating $x$. The number $d_{\mathrm{D}}^{+}(x)=d^{+}(x)=\left|N^{+}(x)\right|$ and $d_{\mathrm{D}}^{-}(x)=$ $d^{-}(x)=\left|N^{-}(x)\right|$ are the outdegree and indegree of $x$, respectively. The minimum outdegree and the minimum indegree of $D$ are denoted by $\delta^{+}(D)=\delta^{+}$ and $\delta^{-}(D)=\delta^{-}$, and $\delta(D)=\delta=\min \left\{\delta^{+}, \delta^{-}\right\}$. The irregularity $I(D)$ of a digraph is $\max \left|d^{+}(x)-d^{-}(y)\right|$ over all vertices $x$ and $y$ of $D(x=y$ is admissible). In addition, the local irregularity is defined by $i_{\ell}(D)=\max \left|d^{+}(x)-d^{-}(x)\right|$ over all vertices $x$ of $D$ and the global irregularity of a digraph $D$ is defined by $i_{\mathrm{g}}(D)=\max \left\{\max \left(d^{+}(x), d^{-}(x)\right)-\min \left(d^{+}(y), d^{-}(y)\right) \mid x, y \in V(D)\right\}$ over all vertices $x$ and $y$ of $D\left(x=y\right.$ is admissible). Clearly, $i_{\ell}(D) \leq I(D) \leq i_{\mathrm{g}}(D)$. If $i_{\mathrm{g}}(D)=0$, then $D$ is regular and if $i_{\mathrm{g}}(D) \leq 1$, then $D$ is almost regular.

A cycle of length $m$ is an $m$-cycle. A cycle or a path in a digraph $D$ is Hamiltonian if it includes all the vertices of $D$. A set $X \subseteq V(D)$ of vertices is independent if the induced subdigraph $D[X]$ has no arcs. The independence number $\alpha(D)=\alpha$ is the maximum size among the independent sets of vertices of $D$.

A digraph $D$ is strongly connected or strong if, for each pair of vertices $u$ and $v$, there is a path from $u$ to $v$ in $D$. A digraph $D$ with at least $k+1$ vertices is $k$-connected if for any set $A$ of at most $k-1$ vertices, the subdigraph $D-A$ obtained by deleting $A$ is strong. The connectivity of $D$, denoted by $k(D)$, is then defined to be the largest value of $k$ such that $D$ is $k$-connected. A set $S$ of vertices of a digraph $D$ is a separating set if $D-S$ is not strong.

If we replace every arc $x y$ of $D$ by $y x$, then we call the resulting digraph, denoted by $D^{-1}$, the converse digraph of $D$.

A digraph $D$ is cycle complementary if there exist two vertex-disjoint cycles $C$ and $C^{\prime}$ such that $V(D)=V(C) \cup V\left(C^{\prime}\right)$.

The following results play important roles in the proof of our main result.

Lemma 1 ([2]). Let $D$ be a c-partite tournament with the partite sets $V_{1}, V_{2}$, $\ldots, V_{\mathrm{c}}$. Then $\| V_{\mathrm{i}}|-| V_{\mathrm{j}}|| \leq 2 I(D) \leq 2 i_{\mathrm{g}}(D)$ for $1 \leq i \leq j \leq c$.

Theorem 1 ([3]). If $D$ is a multipartite tournament, then

$$
k(D) \geq \frac{|V(D)|-2 i_{\ell}(D)-\alpha(D)}{3} .
$$

Theorem 2 ([14]). Let $D$ be a regular 3-partite tournament with $|V(D)| \geq 6$. Then $D$ contains two complementary cycles of length 3 and $|V(D)|-3$, unless $D$ is isomorphic to $D_{3,2}$. ( $D_{3,2}$ is shown in Fig. 1.) 


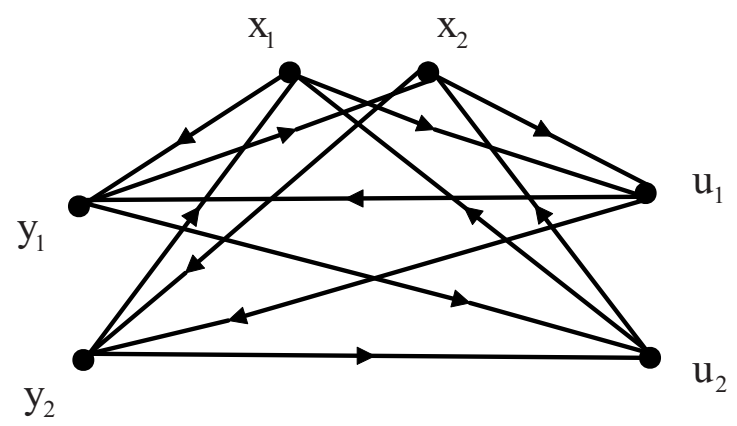

Fig. 1. The 2-regular 3-partite tournament $D_{3,2}$

Theorem 3 ([4]1994). Let $D$ be a strong c-partite tournament with $c \geq 3$. Then at least one vertex of every partite set of $D$ is contained in an $m$-cycle for each $m \in\{3,4, \ldots, c\}$.

Lemma 2 ([2]). If $D$ is an almost regular multipartite tournament, then for every vertex $x$ of $D$ we have

$$
\frac{|V(D)|-\alpha(D)-1}{2} \leq d^{+}(x), d^{-}(x) \leq \frac{|V(D)|-\gamma(D)+1}{2} .
$$

Proposition 1 ([2]). Let $D$ be an almost regular c-partite tournament with the partite sets $V_{1}, V_{2}, \ldots, V_{\mathrm{c}}$ such that $r=\left|V_{1}\right| \leq\left|V_{2}\right| \leq \ldots \leq\left|V_{\mathrm{c}}\right|=r+2$. Then $|V(D)|-r$ is even.

\section{Main Result}

The problem of complementary cycles in tournaments was almost completely solved by Reid [5] in 1985 and by Song [6] in 1993. These authors proved that every 2-connected tournament $D$ on at least 8 vertices has complementary cycles of length $t$ and $|V(D)|-t$ for all $t \in\{3,4, \ldots,|V(D)|-3\}$. Later, Guo and Volkmann [7, 8] extended this result to locally semicomplete digraphs. In addition, there are some results on complementary cycles on bipartite tournaments by Z . Song, K. Zhang, J. Wang and Manoussakis. See [9], [11, [10], 12].

Volkmann 13. confirmed that each regular multipartite tournament is cycle complementary, unless $D$ is isomorphic to $T_{7}, D_{4,2}, D_{4,2}^{*}, D_{3,2}$. But the problem about the existences of complementary cycles in multipartite digraphs which are not tournaments or bipartite tournaments or locally semicomplete digraphs or regular digraphs is still open. It seems that the problem of complementary cycles in semicomplete $n$-partite digraphs with $n \geq 3$ is difficult. In the following, we give the definition of componentwise complementary cycles in multipartite digraphs. 
Definition 1. (componentwise complementary cycles) Let $V_{1}, V_{2}, \ldots, V_{\mathrm{c}}$ be the partite sets of $D$. If there exist two vertex disjoint cycles $C$ and $C^{\prime}$ in $D$ such that $V_{\mathrm{i}} \cap\left(V(C) \cup V\left(C^{\prime}\right)\right) \neq \emptyset$ for all $i=1,2, \ldots, c$, then $C$ and $C^{\prime}$ are two componentwise complementary cycles of $D$.

Clearly, a pair of componentwise complementary cycles of $D$ is a pair of complementary cycles of $D$, if it contains all vertices of $D$.

If $D$ contains a pair of componentwise complementary cycles, then $D$ is cycle componentwise complementary.

Our main result is the following theorem.

Theorem 4. If $D$ is an almost regular 3-partite tournament with $|V(D)| \geq 6$, then $D$ is cycle componentwise complementary, unless $D$ is isomorphic to $D_{3,2}$.

Example 1. Let $V_{1}=\left\{x_{1}, x_{2}\right\}, V_{2}=\left\{y_{1}, y_{2}\right\}, V_{3}=\left\{u_{1}, u_{2}\right\}$ be the partite sets of the 2-regular 3-partite tournament $D_{3,2}$ presented in Fig. 1 . Then it is a simple matter to verify that $D_{3,2}$ contains no componentwise complementary 3-cycles (and of course no complementary cycles).

\section{Proof of Theorem 4}

Let $V_{1}, V_{2}, V_{3}$ be the partite sets of $D$ such that $\left|V_{1}\right| \leq\left|V_{2}\right| \leq\left|V_{3}\right|$. By Theorem1 and $i_{\ell}(D) \leq i_{\mathrm{g}}(D)$,

$$
k(D) \geq \frac{|V(D)|-2 i_{\ell}(D)-\alpha(D)}{3} \geq \frac{|V(D)|-2 i_{\mathrm{g}}(D)-\alpha(D)}{3} .
$$

If $\left|V_{1}\right|=r$, then because of $i_{\mathrm{g}}(D) \leq 1$ we deduce from Lemma 1 that $\left|V_{3}\right|=$ $r+l$ for some $l \in\{0,1,2\}$. Since $|V(D)| \geq 6, r \geq 2$.

Clearly, if $l=0$, then $D$ is a regular 3-partite tournament. By Theorem 2 , $D$ contains a pair of complementary cycles, i.e. $D$ contains a pair of componentwise complementary cycles, unless $D$ is isomorphic to $D_{3,2}$. In the following, we consider the case $l \neq 0$. According to $(1)$, we have $k(D) \geq 1$. In view of Theorem 3 there exists a 3 -cycle $C_{3}$ in $D$. If we define the 3 -partite tournament $H$ by $H=D-V\left(C_{3}\right)$, then $i_{\ell}(H) \leq i_{\mathrm{g}}(H) \leq 3$. We distinguish five cases.

1. $\left|V_{1}\right|=r,\left|V_{2}\right|=r, \quad\left|V_{3}\right|=r+1$.

2. $\left|V_{1}\right|=r,\left|V_{2}\right|=r+1,\left|V_{3}\right|=r+1$.

3. $\left|V_{1}\right|=r,\left|V_{2}\right|=r, \quad\left|V_{3}\right|=r+2$.

4. $\left|V_{1}\right|=r,\left|V_{2}\right|=r+1,\left|V_{3}\right|=r+2$.

5. $\left|V_{1}\right|=r,\left|V_{2}\right|=r+2,\left|V_{3}\right|=r+2$.

According to Proposition 1, we have case 4 is impossible.

Since the proofs of the cases above are similar, we give the proof of case 1 only.

Let $V_{1}=\left\{x_{1}, x_{2}, \ldots, x_{\mathrm{r}}\right\}, V_{2}=\left\{y_{1}, y_{2}, \ldots, y_{\mathrm{r}}\right\}, V_{3}=\left\{u_{1}, u_{2}, \ldots, u_{\mathrm{r}+1}\right\}$, and let $C_{3}=x_{1} y_{1} u_{1} x_{1}$. By Lemma 2 we have $r-1 \leq d_{\mathrm{D}}^{+}(x), d_{\mathrm{D}}^{-}(x) \leq r+1$, for 
$x \in V(D)$. If $H$ contains a cycle $C$, then we have $V\left(C_{3}\right) \cap V(C)=\emptyset$ and $\left(V\left(C_{3}\right) \cup V(C)\right) \cap V_{\mathrm{i}} \neq \emptyset$ for all $i=1,2,3$. Otherwise, let $P=v_{1} v_{2} \ldots v_{\mathrm{p}}$ be a longest path of $H$. Clearly, $v_{1} \Rightarrow V\left(D-V\left(C_{3}\right)\right)$ and $V\left(D-V\left(C_{3}\right)\right) \Rightarrow v_{\mathrm{p}}$. Hence, we have

$$
\begin{gathered}
d_{\mathrm{D}}^{+}\left(v_{1}\right) \geq 3 r+1-3-r=2 r-2, \text { and } \\
d_{\mathrm{D}}^{-}\left(v_{1}\right) \leq 2 .
\end{gathered}
$$

If $r \geq 4$, then $d_{\mathrm{D}}^{+}\left(v_{1}\right) \geq 2 r-2>r+1$. The contradiction to the almost regular of $D$.

If $r=3$, then $d_{\mathrm{D}}^{+}\left(v_{1}\right) \geq 2 r-2$. Since $d_{\mathrm{D}}^{-}\left(v_{1}\right) \leq 2$, we have $d_{\mathrm{D}}^{+}\left(v_{1}\right)-d_{\mathrm{D}}^{-}\left(v_{1}\right) \geq$ $2 r-4=2$, a contradiction to $i_{\mathrm{g}}(D) \leq 1$.

If $r=2$, then $\left|V_{1}\right|=2,\left|V_{2}\right|=2$, and $\left|V_{3}\right|=3$. If $p<3$, i.e. $p=2$, then there is one vertex $u$ such that it is adjacent with $y$ for all $y \in V(P)$. Since $P$ is a longest path in $H, v_{1} \rightarrow u$ and $u \rightarrow v_{2}$. Then $H$ has a path $x_{1} u x_{2}$, a contradiction. Therefore, $p=3$ or $p=4$. If there exists $V_{\mathrm{i}}$ such that $V_{\mathrm{i}} \cap V(P)=\emptyset$ for some $i \in\{1,2,3\}$, then there is a vertex $z \in V_{\mathrm{i}}$ such that it is adjacent with $y$ for all $y \in V(P)$. Since $P$ is a longest path in $H, v_{1} \rightarrow z$ and $z \rightarrow v_{\mathrm{p}}$. Then there is an integer $j \in\{1,2, \ldots, p\}$ such that $v_{\mathrm{j}-1} \rightarrow z \rightarrow v_{\mathrm{j}}$. Therefore, $H$ has a $(p+1)$-path , a contradiction. We consider two cases.

(1) Let $p=3$. Then $P=v_{1} v_{2} v_{3}$.

If $v_{2} \in V_{3}$, then there is one vertex $x \in V_{3}$ such that $v_{1} \rightarrow x \rightarrow v_{3}$. Let, without loss of generality, $v_{1} \in V_{1}$ and $v_{3} \in V_{2}$. Since $i_{\mathrm{g}}(D) \leq 1,\left\{y_{1}, u_{1}\right\} \rightarrow v_{1}$, $v_{3} \rightarrow\left\{x_{1}, u_{1}\right\}$. If $y_{1} \rightarrow v_{2}$, then $v_{2} \rightarrow x_{1}$ and we obtain the componentwise complementary cycles $x_{1} y_{1} v_{2} x_{1}$ and $u_{1} v_{1} x v_{3} u_{1}$. If $v_{2} \rightarrow y_{1}$, then $x_{1} \rightarrow v_{2}$. Furthermore, if $x_{1} \rightarrow x$, then we have the componentwise complementary cycles $x_{1} x v_{3} u_{1} x_{1}$ and $y_{1} v_{1} v_{2} y_{1}$; if $x \rightarrow x_{1}$, then $y_{1} \rightarrow x$ and we obtain the componentwise complementary cycles $x_{1} y_{1} x x_{1}$ and $u_{1} v_{1} v_{2} v_{3} u_{1}$.

Let $v_{3} \in V_{3}$. If $v_{1} \in V_{3}$, then we consider $D^{-1}$. Let, without loss of generality, $v_{1} \in V_{1}$ and $v_{2} \in V_{2}$. Since $P$ is a longest path in $H,\left|V_{1}\right|=2,\left|V_{2}\right|=2$, and $\left|V_{3}\right|=3$, there is one vertex $x \in V_{3}$ such that $\left\{v_{1}, v_{2}\right\} \rightarrow x$. Since $i_{\mathrm{g}}(D) \leq 1$, $\left\{v_{3}, x\right\} \rightarrow\left\{x_{1}, y_{1}\right\},\left\{y_{1}, u_{1}\right\} \rightarrow v_{1}$, and $x_{1} \rightarrow v_{2}$. If $v_{2} \rightarrow u_{1}$, then we have the componentwise complementary cycles $x_{1} v_{2} u_{1} x_{1}$ and $y_{1} v_{1} v_{3} y_{1}$. If $u_{1} \rightarrow v_{2}$, then we have the componentwise complementary cycles $x_{1} v_{2} x x_{1}$ and $y_{1} u_{1} v_{1} v_{3} y_{1}$.

(2) Let $p=4$. Then $P=v_{1} v_{2} v_{3} v_{4}$.

Note that $\left|V_{1}\right|=2,\left|V_{2}\right|=2$, and $\left|V_{3}\right|=3$.

First we assume that $V\left(v_{1}\right)=V\left(v_{4}\right)$, i.e. $V\left(v_{1}\right)=V\left(v_{4}\right)=V_{3}$. Let, without loss of generality, $v_{2} \in V_{1}$ and $v_{3} \in V_{2}$. Since $i_{\mathrm{g}}(D) \leq 1,\left\{x_{1}, y_{1}\right\} \rightarrow v_{1}$ and $v_{4} \rightarrow\left\{x_{1}, y_{1}\right\}$.

If $v_{3} \rightarrow x_{1}$ and $u_{1} \rightarrow v_{2}$, then we obtain the componentwise complementary cycles $x_{1} v_{1} v_{3} x_{1}$ and $y_{1} u_{1} v_{2} v_{4} y_{1}$.

If $v_{3} \rightarrow x_{1}$ and $v_{2} \rightarrow u_{1}$, then we have $y_{1} \rightarrow v_{2}$ and $u_{1} \rightarrow v_{3}$. This leads to the componentwise complementary cycles $y_{1} v_{2} v_{4} y_{1}$ and $x_{1} v_{1} v_{3} x_{1}$.

If $x_{1} \rightarrow v_{3}$, then we have $v_{3} \rightarrow u_{1}$ and $u_{1} \rightarrow v_{2}$. Furthermore, if $v_{2} \rightarrow y_{1}$, then we obtain the componentwise complementary cycles $y_{1} u_{1} v_{2} y_{1}$ and $x_{1} v_{1} v_{3} v_{4} x_{1}$; 
if $y_{1} \rightarrow v_{2}$, then we obtain the componentwise complementary cycles $y_{1} v_{2} v_{4} y_{1}$ and $x_{1} v_{1} v_{3} u_{1} x_{1}$.

Now we assume that $V\left(v_{1}\right) \neq V\left(v_{4}\right)$. Let, without loss of generality, $\left\{v_{2}, v_{4}\right\} \subset$ $V_{3}$. If $\left\{v_{1}, v_{3}\right\} \subset V_{3}$, then we consider $D^{-1}$. Let, without loss of generality, $v_{1} \in V_{1}$ and $v_{3} \in V_{2}$. Since $i_{\mathrm{g}}(D) \leq 1,\left\{y_{1}, u_{1}\right\} \rightarrow v_{1}$ and $v_{4} \rightarrow\left\{x_{1}, y_{1}\right\}$.

If $y_{1} \rightarrow v_{2}$, then we have $v_{2} \rightarrow x_{1}, x_{1} \rightarrow v_{3}$, and $v_{3} \rightarrow u_{1}$. This leads to $x_{1} v_{3} u_{1} x_{1}$ and the componentwise complementary cycle $y_{1} v_{1} v_{4} y_{1}$.

If $v_{2} \rightarrow y_{1}$, then $x_{1} \rightarrow v_{2}$. Furthermore, if $v_{3} \rightarrow u_{1}$, then we obtain the componentwise complementary cycles $u_{1} x_{1} v_{2} v_{3} u_{1}$ and $y_{1} v_{1} v_{4} y_{1}$; if $u_{1} \rightarrow v_{3}$, then $v_{3} \rightarrow x_{1}$ and the componentwise complementary cycles $x_{1} v_{2} v_{3} x_{1}$ and $y_{1} u_{1} v_{1} v_{4} y_{1}$.

The proof is complete.

\section{Acknowledgements}

This work was supported from NSFC under Grant No. 60373025 and the fund of Ludong University under Grant No. 042711. We want to show our appreciation here.

\section{References}

1. J.A.Bondy, Diconnected orientation and a conjecture of Las Vergnas, J. London Math. Soc., 14(1976), 277-282.

2. A.Yeo. Diregular c-partite tournaments are vertex-pancyclic when $c \geq 5$. J. Graph Theory, 32 (1999), 137-152.

3. A.Yeo, Semicomplete multipartite digraphs, Ph.D. thesis, Odense University, 1998.

4. Y. Guo, L. Volkmann. Cycles in multipartite tournaments. J. Combin. Theory Ser.B, 62 (1994), 363-366.

5. K. B. Reid, Two complementary circuits in two-connected tournaments, Ann. Discrete Math., 27(1985): 321-334.

6. Z. M. Song, Complementary cycles of all lengths in tournaments, J. Combin. Theory Ser.B, 57(1993): 18-25.

7. Y. Guo and L. Volkmann, On complementary cycles in locally semicomplete digraphs, Discret Math. 135(1994), 121-127.

8. Y. Guo and L. Volkmann, Locally semicomplete digraphs thar are complementary m-pancyclic, J. Graph Theory 21 (1996), 121-136.

9. Z. Song, Complementary cycles in bipartite tournaments, J. Nanjing Inst. Tech. 18(1988): 32-38.

10. K. Zhang, Y. Manoussakis, and Z. Song, Complementary cycles containing a fixed arc in diregular bipartite tournaments, Discrete Math. 133 (1994), 325-328.

11. K. Zhang and Z. Song, Complementary cycles containing a pair of fixed vertices in bipartite tournaments, Appl. J. Chin. Univ. 3 (1988), 401-407.

12. K. Zhang and J. Wang, Complementary cycles containing a fixed arc and a fixed vertex in bipartite tournaments, Ars Combin. 35 (1993), 265-269.

13. L. Volkmann, Complementary cycles in regualr multipartite tournaments, where one cycle has length four, Kyungpook Math., J., 44(2004), 219-247.

14. L. Volkmann, All regualr multipartite tournaments that are cycle complementary, Discrete Math., 281(2004), 255-266. 